\title{
Microcracks and mechanical behaviour of corio-epidermal junction of equine hoof
}

\author{
Petra Kochová ${ }^{1, a}$, Zbyněk Tonar ${ }^{1,2, b}$, Kirsti Witter ${ }^{3, \mathrm{c}}$ and Jana Mezerová ${ }^{4, \mathrm{~d}}$ \\ ${ }^{1}$ Department of Mechanics, Faculty of Applied Sciences, University of West Bohemia, Univerzitní \\ 22, 30614 Pilsen, Czech Republic \\ ${ }^{2}$ Institute of Histology and Embryology, Faculty of Medicine in Pilsen, Charles University in Prague, \\ Karlovarská 48, 30166 Pilsen, Czech Republic \\ ${ }^{3}$ Institute of Histology and Embryology, Department of Pathobiology, University of Veterinary Medic \\ ine Vienna, Veterinärplatz 1, A-1210 Vienna, Austria \\ ${ }^{4}$ Veterinary Clinic Heřmanův Městec, Nový Dvůr 993, 53803 Heřmanův Městec, Czech Republic

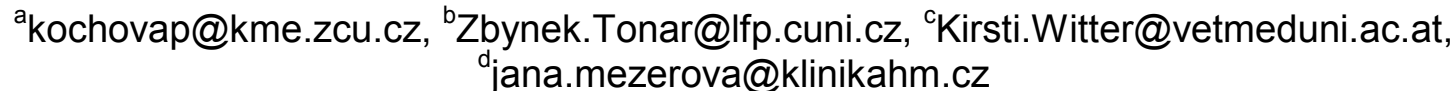

Keywords: Mechanical measurement; Stiffness evaluation

\begin{abstract}
The corio-epidermal junction (CEJ) of equine hoof is highly vascularized, tough, but flexible suspensory apparatus between the wall of the hoof and the third distal phalanx. The connection created by leaf-like lamellae has a key role in the solidity and health of the hoof. In this study we focus on mechanical behaviour of CEJ under tensile loading and on the origin and spatial spreading of cracks of CEJ produced by stress exceeding the ultimate strength of the junction. The results show visible relation between mechanical results and structural parameters: the probability of rupture origin is higher in the case of small values of Young $s$ moduli of elasticity in region with small deformations and small values of 2D length density in dermal region.
\end{abstract}

\section{Introduction}

The corio-epidermal junction (CEJ) is a highly vascularized dermo-epidermal layer located between the walls and the third distal phalanx of equine hoof. This very strong and resilient interdigitation between dermal (near distal phalanx) and epidermal (near wall horn) tissue is composed of secondary lamellae (SL) which cover the primary lamellae (PL) of corium. These 500-600 parallel leaf-like PL covered by 150-200 SL along the length of each of the PL of CEJ enable supporting the $400 \mathrm{~kg}$ of a horse and have a key role in the strength and the health of the hoof. The main connecting component of CEJ is the extracellular basement membrane. The basement membrane forms the structural boundary between vascular dermal and avascular epidermal compartments [1].

Aims. Although CEJ, e.g. its interlaminar spacing and laminar orientation, was previously described in detail in many studies $[1,2,3]$, there is a lack of quantitative information about relation between mechanical properties and microstructure of this junction. We continue in the detailed quantification of CEJ of equine hoof started earlier [4]. Here we focus on quantification of rupture induced by mechanical loading up to ultimate strength, especially on connection between mechanical properties, length densities of SL, rupture origin and rupture spreading. Moreover, the basement membrane is known to be a tough unbroken sheet of extracellular matrix. From this point of view it was interesting to elucidate whether the rupture will break the basement membrane or it will go along this strong connection.

Our aim was to verify the following hypotheses:

- $\mathrm{H}_{1}$ : The length density of SL does not influence the origin of CEJ rupture.

- $\mathrm{H}_{2}$ : The ruptures prefer neither dermal nor epidermal region.

- $\mathrm{H}_{3}$ : The rupture is going through basement membrane.

- $\mathrm{H}_{4}$ : The length density of SL does not influence the Young S moduli of elasticity of CEJ. 


\section{Material and Methods}

For quantitative microscopy as well as mechanical measurement we took two equine hoofs from a single adult horse. The specimens from one hoof were stored as frozen before measurement and the specimens from the second hoof were fixed using formalin. We took always three specimens (I, II, III) from each region (front (F) and quarter (lateral part of the foot, Q) from the left (L) and the right (R) side, as illustrated in Fig.1 left) of the hoof, so we finally had twelve specimens per one hoof. Each specimen was cut into two halves. One half was exposed to mechanical loading and the second half underwent histological processing and was used for quantitative microscopy to obtain a reference state of microstructure before possible rupture.
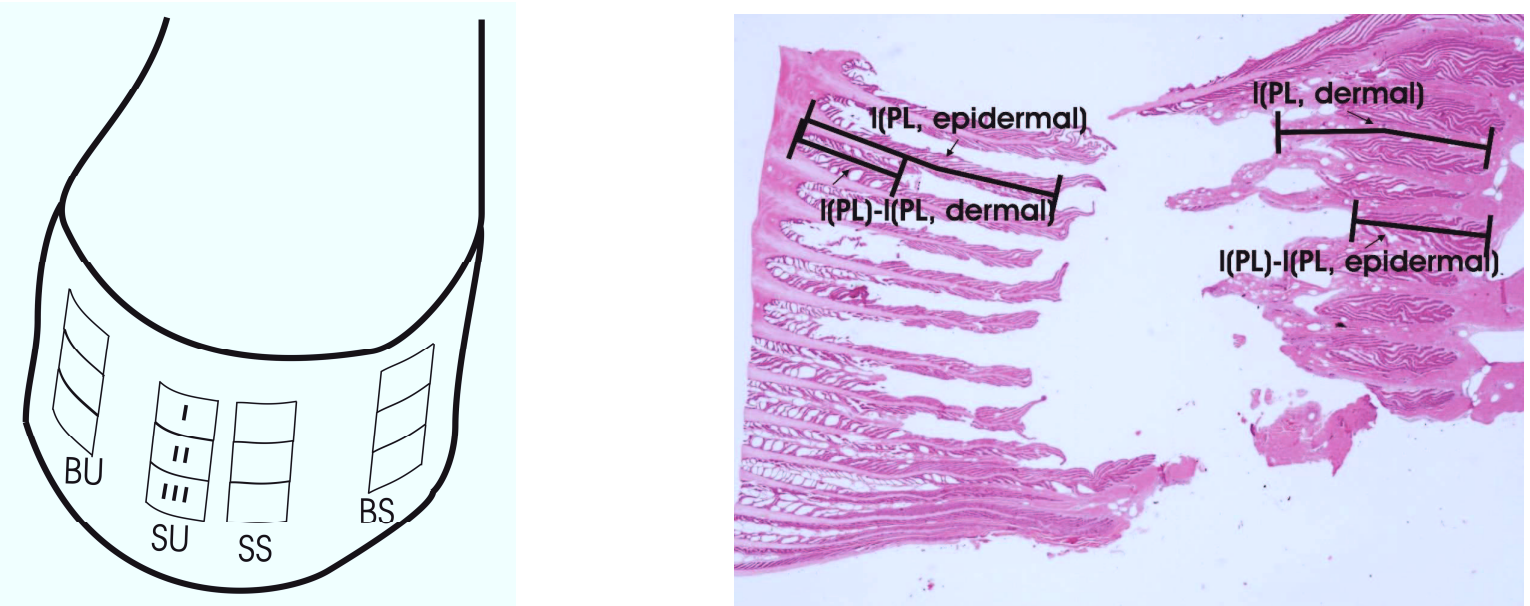

Fig.1: Left: Location of specimens taken from hoof for mechanical measurement and quantitative microscopy: F - front, Q - quarter (lateral part of the foot), L - left, R $\square$ right, I, II, III $\square$ numbers of specimens from each region (numbered in proximodistal order). Right: A scheme of evaluated parameters used for rupture quantification: $l(P L)$ - length of CEJ of intact tissue, $l(P L$, dermal) and $l(P L$, epidermal) - lengths of a pieces of the PL in dermal and epidermal region, respectively.

Quantitative Microscopy. In order to quantify the rupture of CEJ we estimated and measured following quantitative parameters: $2 \mathrm{D}$ length density of SL in dermal $L_{A}(S L$, dermal) as well as in epidermal region $L_{A}(S L$, epidermal), length of the PL $l(P L)$ in the intact tissue; length of pieces of the primary dermal $l(P L$, dermal) and epidermal $l(P L$, epidermal) lamellae after rupture, length of the rupture l(rupture) and number of intersections between basement membrane and the rupture $P_{L}$. For quantification we used the stereological methods integrated in Ellipse (ViDiTo, SR) and ImageJ (Wayne Rasband, National Institutes of Health, USA).

The $2 \mathrm{D}$ length density $L_{A}(S L$, dermal/epidermal) was assessed using a method based on counting the intersections of the basement membrane and a network of circular arcs that was randomly superposed on the micrographs:

$L_{A}(S L$, dermal/epidermal $)=\left(\frac{\pi}{2} \cdot \sum Q_{i}\right) /\left((l / p) \cdot \sum P_{i}\right)$,

where $l / p$ is the length of a circular arc of the test system, $\Sigma Q_{i}$ is the number of intersections between the system of circular arcs used and the basement membrane of SL and $\Sigma P_{i}$ is the total number of circular arcs in the reference region.

To quantify if the ruptures do not prefer the dermal or epidermal region (hypothesis $\mathrm{H}_{2}$ ) we counted the parameters $\Delta l(P L$,dermal) and $\Delta l(P L$,epidermal) which present the length of the PL remained in epidermal and dermal region after rupture, given by:

$\Delta l(P L$, dermal $)=\frac{l(P L)-l(P L, \text { epidermal })}{l(P L)}, \Delta l(P L$, epidermal $)=\frac{l(P L)-l(P L, \text { dermal })}{l(P L)}$, 
where $l(P L$, dermal $), l(P L$, epidermal $)$ are the lengths of the pieces of the primary dermal and epidermal lamellae of ruptured tissue and $l(P L)$ is the length of CEJ of intact tissue, Fig.1 right.

To quantify the microcracks (hypothesis $\mathrm{H}_{3}$ ), i.e. if the rupture broke the basement membrane or not we took five micrographs from each rupture using systematic uniform random sampling to cover whole specimen. From these micrographs we took four regions. So we finally had twenty regions used for quantification. We counted the theoretical number of intersections $P \llbracket$ between rupture profiles and basement membrane given by:

$P_{L}^{\prime}=l($ rupture $) \cdot \frac{2}{\pi} \cdot L_{A}(S L$, dermal / epidermal $)$,

where $L_{A}(S L$, dermal/epidermal) is the 2D length density of SL and l(rupture) is the length of the rupture.

Mechanical Measurement. The specimens (about $20 \mathrm{~mm}$ in length, $10 \mathrm{~mm}$ in thickness and $6 \mathrm{~mm}$ in width) were clamped into the pneumatic grips of a traction machine (Zwick/Roell GmbH $\&$ Co, Germany) in such way that way the bone of the third distal phalanx and the wall were held in the jaws of testing machine so that only CEJ was exposed to mechanical loading. Following the previous study [4] the specimens were firstly preconditioned (exposed to a cyclic loading with small deformations). The cyclic loading involved 50 cycles, one cycle consisted of a linearly growing elongation up to strain $18 \%$ and a symmetrical shortening back to the initial length of each specimen. The loading velocity was $500 \mathrm{~mm} / \mathrm{min}$ for both parts of the cycle. This elongation rate corresponds to the velocity that is produced by equine gallop [3]. After this preconditioning, the tensile test with a linearly growing elongation up to tissue rupture was applied. The Young $\mathrm{S}$ modulus of elasticity in region of small deformations (toe region) and region of large deformations (linear region) and the ultimate stress and strain were all determined using our software for experimental lab fitting elfpy [5].

Statistical Analysis. The Statistica base 9.1 (Statsoft, Inc., Tulsa, OK, USA) was applied to the results of quantitative microscopy and mechanical measurements. The normality of the data was tested with the Shapiro-Wilk $₫ \mathrm{~S}$ test. The correlations between Young $\mathrm{s}$ moduli of elasticity in toe and linear region, ultimate strain and stress and the structural parameters (hypotheses $\mathrm{H}_{1}, \mathrm{H}_{4}$ ) were determined by Spearman Rank Order Correlation test. The differences between theoretical and real number of intersections between rupture and basement membrane were tested using parametric ttest (hypothesis $\left.\mathrm{H}_{3}\right)$. Differences between $\Delta l\left(P L\right.$,dermal) and $\Delta l\left(P L\right.$,epidermal) (hypothesis $\left.\mathrm{H}_{2}\right)$ were tested using Wilcoxon matched pairs test. All resultant values are presented as means \pm standard deviations.

\section{Results and Discussion}

Quantitative Microscopy. The mean 2D length density of SL was $L_{A}(S L$ dermal/epidermal $)=$ $0.027 \pm 0.06 \mu^{-1}$. The difference between $\Delta l(P L$,dermal) and $\Delta l(P L$,epidermal) was not significant $(\mathrm{p}=0.07)$, although the value $\Delta l(P L$, dermal $)=0.29$ was lower than $\Delta l(P L$,epidermal $)=0.45$, i.e. the ruptures preferred neither dermal nor epidermal region.

Further, we found no significant difference $(\mathrm{p}=0.985)$ between values of counted and real number of intersections between rupture and basement membrane $\left(P_{L}=15.23 \pm 8.85, P \square_{L}=15.27 \pm 8.36\right)$. In other words the microcracks did go randomly and did not prefer the region along basement membrane or basement membrane alone.

Otherwise, the results showed that an increase of $L_{A}(S L$, dermal) was connected with a decrease of $\Delta l(P L$,dermal) (Spearman $\mathrm{R}=-0.70)$. Thus, the rupture originating in the dermal region occurred in the part with lower 2D length density of SL. In other words the higher value of length density of SL in dermal region is connected with lower probability of total rupture in this region. There was also visible positive correlation between $2 \mathrm{D}$ length densities of SL in dermal and epidermal regions (Spearman $\mathrm{R}=0.44)$. The increase in value of the $L_{A}(S L$, epidermal) was connected with lower probability that the rupture occurs (Spearman $\mathrm{R}=0.47$ ). 
Mechanical Measurement. From all tested specimens (twenty two, twelve per each equine hoof) there were eight totally ruptured, three partially ruptured and eleven specimens without rupture (especially in the case of fixed specimens) visible on microstructural level. The value of Young $\mathrm{s}$ modulus of elasticity in region of small deformations was $\mathrm{E}_{\text {toe }}=1.05 \pm 1.33 \mathrm{MPa}$ and the value of Young $\mathrm{S}$ modulus of elasticity in linear region was $\mathrm{E}_{\text {linear }}=11.07 \pm 6.74$. The value of ultimate strain was $0.54 \pm 0.21$ and the value of ultimate stress was $2.95 \pm 1.35 \mathrm{MPa}$.

An increase in Young $\mathrm{S}$ modulus of elasticity in linear region was connected with increase of ultimate stress (Spearman $\mathrm{R}=0.57$ ) and decrease of ultimate strain (Spearman $\mathrm{R}=-0.45$ ). An increase of the value in toe region was connected with increase of Young $\mathrm{S}$ moduli of elasticity in linear region (Spearman $\mathrm{R}=0.56$ ) and decrease of ultimate strain (Spearman $\mathrm{R}=0.61$ ). The results showed that when the tissue was stiff in the toe region, it had the higher stiffness in region of large deformations too and it was less extensible than the tissue with lower stiffness in toe region.

Correlation between Results of Mechanical Measurement and Quantitative Microscopy. A positive correlation was found between $L_{A}(S L$, dermal) (Spearman $\mathrm{R}=0.61)$ as well as $L_{A}(S L$, epidermal) (Spearman $\mathrm{R}=0.46$ ) and Young $\mathrm{S}$ modulus of elasticity in region of small deformations ( $\left.\mathrm{E}_{\text {toe }}\right)$. The increase of $L_{A}(S L$, epidermal) was also connected with decrease of ultimate strain (Spearman $\mathrm{R}=-0.47$ ). The Young $\mathrm{S}$ modulus of elasticity in toe region was connected with parameter $\Delta l(P L$,dermal) in negative correlation (Spearman $\mathrm{R}=-0.64)$. An increase of $2 \mathrm{D}$ length density of SL in epidermal region (Spearman $\mathrm{R}=0.47$ ) and an increase of Young $\mathrm{S}$ modulus of elasticity in toe region (Spearman $\mathrm{R}=0.53$ ) were connected with lower probability of origin of a tissue rupture.

Thus, it was visible, that the $2 \mathrm{D}$ density influences in the first place the Young $\mathrm{s}$ modulus of elasticity in toe region, i.e. region with small deformation. In the physiological conditions there are just small deformations which occur in CEJ. It was visible, that the small value of Young $\mathrm{s}$ modulus of elasticity in toe region lead to higher probability of rupture origin.

\section{Summary}

The complex mechanical and microscopic study of CEJ was done. The relation between mechanical results and structural parameters was visible: the probability of rupture origin was higher in the case of small values of Young $s$ moduli of elasticity in toe region and small values of $2 \mathrm{D}$ length density in dermal region. Thus we contradicted hypothesis $\mathrm{H}_{1}$ and $\mathrm{H}_{4}$. The stiffer specimens reached higher values of ultimate stress connected with smaller values of ultimate strain when compared to the less stiff specimens. Further, we accepted the hypothesis $\mathrm{H}_{2}$ that the rupture prefer neither dermal nor epidermal region and contradicted hypothesis $\mathrm{H}_{3}$ that the rupture in going through basement membrane.

Acknowledgments. The study was supported by the GACR projects 106/09/734 and 106/09/P226 and research project MSM 4977751303.

\section{References}

[1] J. J. Thomason, H. L. McClinchey, B. Faramarzi and J. C. Jofriet: The anatomical records part A. Vol. 283A (2005), p. 366 379.

[2] J. J. Thomason, H. L. McClinchey, B. Faramarzi and J. C. Jofriet: The anatomical records part A. Vol. 283A (2005), p. 366 379 .

[3] J. E. Douglas, T. L. Biddick, J. J. Thomason and J. C. Jofriet: J Exp Biol. Vol. 201 (1998).

[4] P. Kochová, Z. Tonar, K. Witter and J. Mezerová: Plzeňský lékařský sborník, in press.

[5] elfpy, http://code.google.com/p/elfpy/ (2010). 